\title{
BMJ Open A comparison of effectiveness between oral rapid testing and routine serum- based testing for HIV in an outpatient dental clinic in Yuxi Prefecture, China: a case-control study
}

\author{
Shifu Li, ${ }^{1}$ Shu Su, ${ }^{2}$ Shunxiang Li, ${ }^{1}$ Liangmin Gao, ${ }^{1,3}$ Ying Cai, ${ }^{1}$ Jincui Fu, ${ }^{1}$ \\ Chunyuan Guo, ${ }^{1}$ Wei Lu, ${ }^{4}$ Feng Cheng, ${ }^{3}$ Jun Jing, ${ }^{3}$ Liang Chen, ${ }^{1}$ Lei Zhang ${ }^{2,3,5,6}$
}

To cite: Li S, Su S, Li S, et al. A comparison of effectiveness between oral rapid testing and routine serum-based testing for HIV in an outpatient dental clinic in Yuxi Prefecture, China: a case-control study. BMJ Open 2017;7:e014601. doi:10.1136/ bmjopen-2016-014601

- Prepublication history and additional material are available. To view these files please visit the journal online (http://dx.doi.org/ 10.1136/ bmjopen-2016-014601)

FC and LZ contributed equally.

Received 10 October 2016 Revised 14 February 2017 Accepted 10 March 2017

CrossMark

For numbered affiliations see end of article.

\section{Correspondence to}

Feng Cheng; feng.cheng@ biomed.tsinghua.edu.cn and $\mathrm{Dr}$ Lei Zhang; lzhang@kirby.unsw. edu.au

\section{ABSTRACT}

Objective To compare the outcomes of routine providerinitiated HIV testing and counselling (PITC) and oral rapid HIV testing for dental clinic outpatients in a hospital. Design We employed a case-control study design and recruited dental outpatients into routine serum-based and oral rapid testing groups. We compared the acceptance, completion and result notification rate between groups. Setting A dental outpatient clinic in the Yuxi People's Hospital, Yunnan.

Participants A total of 758 and 816 dental outpatients were enrolled for routine and oral rapid testing, respectively.

Results The percentage of participants willing to receive routine HIV testing was $28.1 \%$ (95\% Cl $24.9 \%$ to $31.3 \%)$ and $96.1 \%(95 \% \mathrm{Cl} 94.8 \%$ to $97.4 \%, \chi 2=186.4, \mathrm{p}<0.001)$ for the rapid testing. Among accepted participants, the percentage of participants who received HIV testing was $26.8 \%$ (95\% Cl $20.9 \%$ to $32.7 \%$ ) in the routine testing group and $100.0 \%$ in the oral rapid HIV testing group $(\chi 2=77.5, p<0.001)$. About $93.0 \%$ of routine testers returned for the test results on the next day, whereas all rapid testers received their test results on the same day $(\chi 2=34.6, p<0.001)$. These correspond to an overall completion rate of $7.0 \%(95 \% \mathrm{Cl} 5.2 \%$ to $8.8 \%$ ) and $96.1 \%$ (95\% Cl $94.8 \%$ to $97.4 \%, \mathrm{p}<0.001)$, respectively. Among the 545 patients who declined routine serum-based HIV testing, the main reasons included, an unnecessary hassle $(254 / 545,46.6 \%)$, having been previously tested $(124 / 545,22.8 \%)$ and self-perceived low risk of HIV infection (103/545, 18.9\%). In contrast, only 32 individuals declined oral rapid testing, and having received a previous test was the primary reason. Three patients in the rapid testing group were later confirmed HIV-positive, yielding an HIV prevalence of $0.38 \%$.

Conclusion Oral rapid HIV testing is a feasible and efficient approach in a clinical setting.

\section{BACKGROUND}

The HIV epidemic is a concentrated epidemic in China. HIV infection is prevalent among specific high-risk groups, such as
Strengths and limitations of this study

- A study that comprehensively compares the strengths and weaknesses of routine serum-based and oral rapid testing methods in a clinical setting.

- It proposed a feasible model to provide rapid HIV screening for outpatients in a developing country setting.

- It explored the underlying reasons for people declining HIV testing.

- A potential selection bias may exist in the study since participants were recruited primarily during daytime hours on weekdays.

- It is uncertain if all nurses and physicians complied with the study protocol throughout the two study phases.

- Participants may feel 'obliged' to participate in the HIV testing to avoid upsetting the clinicians.

- The study was conducted in only one clinic which may bring potential biases; multiple research settings could be considered in future studies.

men who have sex with men, injecting drug users and female sex workers. ${ }^{1}$ In response to UNAIDS 90-90-90 goals, HIV testing has been substantially scaled-up in China to prevent HIV from further spreading to the general population. ${ }^{2}$ Regular HIV testing has already been included in routine care for pregnant women attending antenatal care and patients before surgery nationally. In jurisdictions where HIV prevalence among the general population is $>1 \%$, routine HIV testing is also recommended in hospitals. ${ }^{3}$ Integration of HIV testing into routine clinical care in hospitals has been shown to be a practical and efficient way for identifying HIV-infected individuals who have not been screened previously. ${ }^{4}$ Routine provider-initiated HIV testing and counselling (PITC) has become routine since 2013 for outpatients 
who attend regular physical check-up in public hospitals in Yuxi County, Southwest China.

PITC employs an 'opt-out' approach, ${ }^{5}$ which is a streamlined model recommended by the World Health Organization (WHO) and the Joint United Nations Programme on HIV/AIDS (UNAIDS) to increase the opportunities for diagnosing HIV in health facilities. ${ }^{6}$ PITC is often included routinely as a part of standard medical care by the clinician. Testing remains voluntary, and the patient has the right to decline HIV referral service and testing. ${ }^{5}$ Past studies have documented that PITC can significantly improve timely HIV diagnosis and treatment, leading to a reduction in HIV-related morbidity and mortality. ${ }^{78}$ Counselling sessions and awareness of one's own HIV status help to reduce risky sexual practices, especially among HIV serodiscordant couples, and hence reduce the transmission of HIV. ${ }^{9}{ }^{10}$ PITC has a number of shortcomings, including low acceptance rate, ${ }^{11}$ high operating cost, concerns about confidentiality and creditability of screening. ${ }^{12-14}$ HIV-related stigma at both individual and structural levels is also a significant barrier preventing people from HIV screening. ${ }^{6}$ PITC has been criticised from an ethical perspective for neglecting to obtain explicit informed consent from patients. ${ }^{15} 16$ Individual factors, such as fear, denial and low-risk perception among patients, as well as service provider-related factors, such as concerns about the added costs to hospital services, increasing workload and care commitment for newly diagnosed HIV-infected patients, contribute to the barriers for implementing PITC. ${ }^{17}{ }^{18}$ Additionally, patients who have difficulty in understanding PITC consent and eligibility assessment are often excluded from the service. ${ }^{1920}$

Past studies have shown that reducing the time required for the testing and counselling procedures improves people's willingness to participate. ${ }^{21} 22$ Studies from both developed and developing countries, such as the USA, Canada, UK, Australia, India and South Korea have demonstrated the benefits of the oral rapid HIV testing method compared with routine serum-based testing. ${ }^{23-29}$ The oral fluid-based test for HIV in India, in particular, is shown to perform better than the finger stick test, to be accurate and well accepted by hospital participants. ${ }^{24} 30$ More recently, two independent studies on oral rapid HIV testing have been conducted in China ${ }^{31}{ }^{32}$ and demonstrated a high acceptance of oral HIV testing in dentists and willingness to integrate the testing procedure into their clinical practice. Yunnan province in China has a large number of ethnic minorities and is a major route for illicit drug trafficking in the country. As a result, HIV prevalence is far higher than in other Chinese regions. ${ }^{233}$ The number of people living with HIV in Yunnan province was 79915 (1.70\%o of total population) by the end of October 2014, accounting for about one-sixth of the people living with HIV/AIDS (PLHIV) in the country. ${ }^{34}$ As nearly half of PLHIV remain undiagnosed in China, ${ }^{35}$ Yunnan potentially has the largest number of unidentified HIV cases, suggesting an urgency for effective scale-up of HIV testing in the general population. Given the heterogeneous population in Yunnan, ${ }^{36}{ }^{37}$ previous findings in other parts of China may not be representative here. This study is necessary to identify the effects of oral rapid HIV testing in this local setting.

Yuxi, with 2.3 million residents, is the third-largest city in Yunnan province in Southwest China. The city is located $100 \mathrm{~km}$ south of Kunming, the capital of Yunnan prefecture, and approximately $300 \mathrm{~km}$ north of Vietnam. ${ }^{33} \mathrm{In}$ this study, we examine an improved model of PITC by offering oral rapid HIV testing instead of routine serumbased testing to outpatients at a local dental clinic in Yuxi. We aim to investigate whether this approach might improve HIV testing acceptance rates and result notification rates, and streamline testing procedures. We conduct a case-control study to compare the screening acceptance, completion and result notification rates between the routine and oral rapid HIV testing procedures. It also determines the HIV seroprevalence in the outpatient sample.

\section{MATERIALS AND METHODS}

\section{Study design}

This case-control study was conducted at a dental clinic in the Yuxi People's Hospital. The hospital patients were from a diverse socioeconomic and ethnic background, and hence the collected sample is representative of the local general population in Southwest China. The study was conducted in two consecutive phases, for routine serum-based HIV testing and oral rapid HIV testing, respectively. We designed the study in this way for a number of reasons. We had only one venue for this exploratory study, so we could not conduct the same study in parallel in two clinical settings for comparison. Additionally, heterogeneity might have been introduced if two clinics were chosen. We considered other possibilities: such as randomising the type of HIV testing the patients were to receive. However, as the patients shared the same waiting area, it is hard to approach each patient privately, which means patients might develop a preference based on previous patients' decisions. Additionally, randomisation might also create confusions for nurses and doctors who would have to remember to which group each patient belonged and conduct the appropriate procedure. Based on these considerations, we decided to conduct testing in two separate phases.

During the first phase (April-June 2014), attending patients were encouraged to receive the routine PITC testing procedure. This was then followed by the oral rapid testing approach in the second phase during July and September 2014. The overall study population was based on those patients participating in both phases of the study. As the initial symptoms of AIDS often include oral candidiasis, herpes zoster, herpes simplex, oral bacteria or fungal infection, and recurrent oral ulcer, we chose a dental clinic as our study site for a greater chance of diagnosing HIV-infected patients. 
A

Routine PITC
B

Oral rapid HIV screening
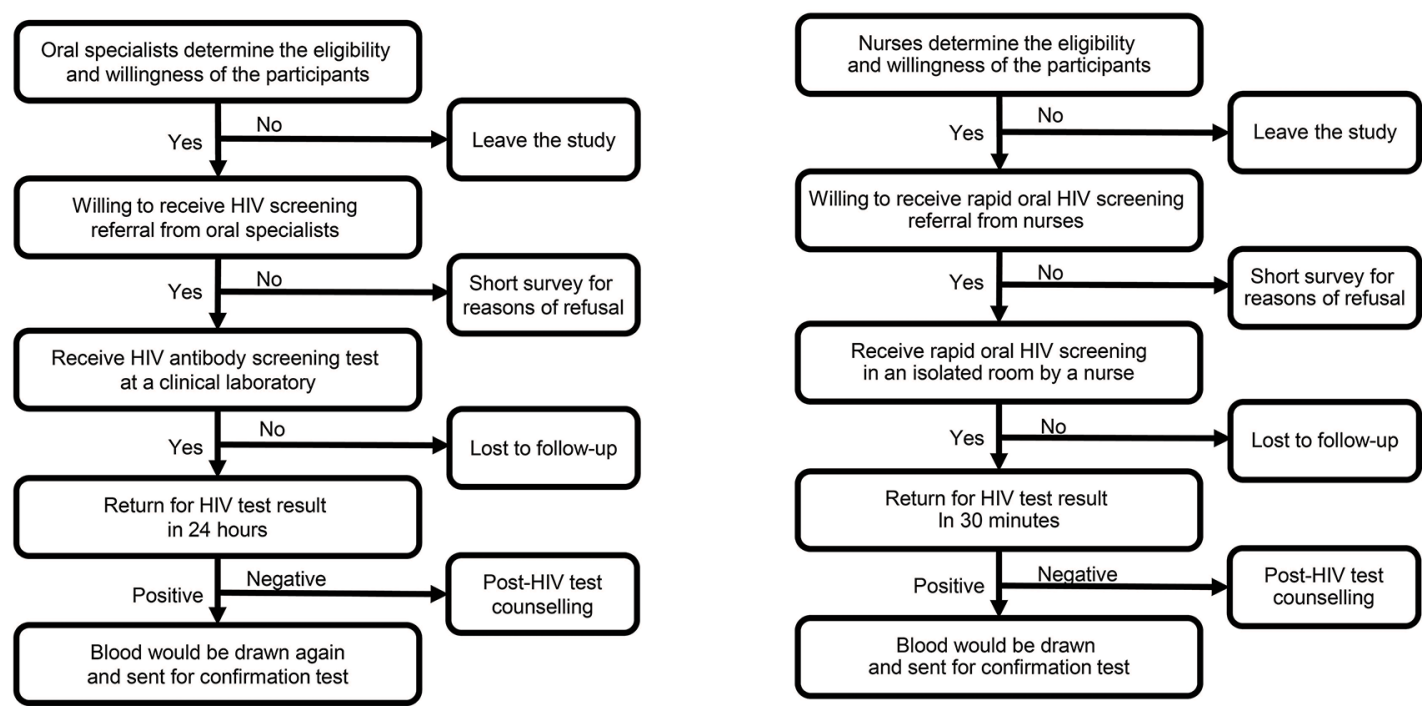

Figure 1 Implementation flowchart for (A) routine provider-initiated HIV testing and counselling (PITC); and (B) oral rapid HIV screening.

Patients who were aged $\geq 18$ years and able to complete a general consent form for care were included in this study. Patients who could not understand the doctor's explanation were excluded. Demographic information of patients, including gender, age, disposable income per month and education level, was recorded. Details of diagnosed oral diseases and HIV infection status were obtained during the doctor consultation and testing.

Figure 1 shows the flow of both routine and rapid testing procedures. In the routine PITC phase, oral doctors determined the eligibility and willingness of the participants and their registered demographic characteristics, and then offered them an HIV referral service. The doctor read the following script to the patients: 'We would like to offer you serum-based HIV testing after your oral treatment, the test will need to draw blood from you, and it will take 1 day for you to receive the test result. You will pay $20 \mathrm{RMB}(\approx \mathrm{US} \$$ 3.3) for this, and your overall oral diseases treatment fee will thereby be increased. However, if you do not want to participate, please sign here'. Patients who declined the referral service completed a separate short survey, and their treatment was not delayed. If patients accepted, a reference sheet was then issued. Patients first received their oral diseases consultation and treatment. Then, patients' blood was collected and tested in the clinical laboratory. Patients were asked to return for their results 24 hours later. Participants who did not receive HIV testing after oral treatment or did not return for their HIV test results were regarded as lost to follow-up. If the testing result was negative, a post-test counselling session was provided. If positive, patients were notified immediately, and another blood sample was drawn for confirmation by western blot assays. The process took about 1 week.
In the rapid HIV phase, HIV testing was also offered by dental doctors. They determined the eligibility of the patients and obtained their registered demographic characteristics, and then offered an HIV referral service using the following script. 'We will offer you oral HIV testing in a separate room before your dental treatment; it will only take you 5 minutes to complete a saliva HIV test (oral mucosal transudate, in case patients could not understand). It is not necessary to draw blood, you will pay $20 \mathrm{RMB}$ for this test, and your overall oral treatment fee will thereby be increased. However, if you do not wish to participate, please sign here'. Patients who refused the HIV testing then completed a separate short survey and their dental treatment was not delayed. If patients accepted, the oral test was performed by a nurse in a separate, private room using oral fluid antihuman immunodeficiency virus (HIV1 +2) antibody diagnostic kit, which is a colloidal gold device (Beijing Marr Bio-Pharmaceutical Co, Ltd) using an oral mucosal swab. ${ }^{24}$ Nurses had been trained by Centers for Disease Control staff in advance to ensure quality. Diagnostic results were available within 20-40 min. Participants who did not return for the test result were regarded as lost to follow-up. If oral screening was negative, a post-test counselling session was provided by a nurse. Otherwise, a blood sample was obtained immediately from the participant and sent for confirmatory testing by western blot assays. Finally, the nurse asked the patients if they were willing to pay 80 RMB for an oral rapid HIV test service (the price was estimated by the testing kit manufacturer and the hospital based on expert opinions) to assess whether participants could afford it. The study was approved by the Tsinghua Universityresearch ethics committee (project ID 20161215). 


\section{Data collection and analysis}

All patients were required to complete a questionnaire when they agreed to participate. The demographic information included the following characteristics: sex, age, disposable income per month, education level and reasons for consultation. Patient's oral and HIV serological examination results were also entered into the database when they became available. If a patient self-reported that they were HIV-infected already, the medical record in the hospital was checked and confirmed. Patients who declined to participate were asked to complete a separate short survey, which specifically asked about reasons for refusal. All information was then transcribed into an Excel spreadsheet (Microsoft Excel 2003, Microsoft Corporation). Data of all surveyed patients were analysed with the Statistical Package for the Social Sciences (SPSS) software version 17.0 for Windows (SPSS Inc, Chicago, Ilinois, USA). A $\chi 2$ test and Fisher's exact test were conducted to investigate the differences among studied variables between the routine and oral rapid HIV testing groups. A p value $<0.05$ was considered significant.

\section{RESULTS}

A total of 1574 patients (639 male, 935 female) participated in this study. Most participants were in the 18-29 year $(34.3 \%)$ age group, had a monthly income higher than 1500 RMB (65.4\%) and had completed high school $(44.5 \%)$. The vast majority of participants visited the dental clinic outpatients owing to common oral or dental issues $(95.8 \%)$ while only $4.2 \%$ of visits were due to opportunistic oral infections. There were no significant differences in demographic characteristics between the routine (758) and oral rapid testing groups (816), and hence the two groups were comparable (table 1).

The oral rapid testing group demonstrated substantially better patient response rate than the routine PITC group in all three indicators (figure 2). In particular, $96.1 \%(784 / 816,95 \%$ CI $94.8 \%$ to $97.4 \%)$ of patients were willing to be screened for HIV in the oral rapid testing group, significantly higher than that of the routine PITC group (213/758, 28.1\%, 95\% CI $24.9 \%$ to $31.3 \%$; $\chi 2=186.4, p<0.001)$. Furthermore, all participants in the rapid HIV testing group who indicated their willingness for HIV screening received it $(784 / 784,100.0 \%)$, but the percentage was only $26.8 \%(57 / 213,95 \%$ CI $20.9 \%$ to $32.7 \%)$ in the routine PITC group $(\chi 2=77.5, \mathrm{p}<0.01)$. Further, all tested patients in the oral rapid testing group received their screening results $(784 / 784,100.0 \%)$. This is significantly higher than the $93.0 \%(53 / 57,95 \%$ CI $86.4 \%$ to $99.6 \%)$ of the tested population in the routine group $(\chi 2=34.6, p<0.01)$. Overall, $96.1 \%$ (784/816, $95 \%$ CI $94.8 \%$ to $97.4 \%$ ) oral rapid testing patients completed the whole screening test and were notified of their results, whereas only $7.0 \%$ (53/758, 95\% CI $5.2 \%$ to $8.8 \%)$ of

Table 1 Key demographic profiles of eligible participants in the study, stratified by group of routine provider-initiated HIV testing and counselling (PITC) and oral rapid HIV testing

\begin{tabular}{|c|c|c|c|c|}
\hline Demographic profile & Total $(n=1574)$ & $\begin{array}{l}\text { Routine PITC }(\mathrm{n}=758) \\
\mathrm{n}(\%)\end{array}$ & $\begin{array}{l}\text { Oral rapid HIV testing }(n=816) \\
n(\%)\end{array}$ & $\chi 2$ Test, $p$ value \\
\hline Sex & & & & $<0.001,0.509$ \\
\hline Female & 935 & $450(59.4)$ & $485(59.4)$ & \\
\hline Age (years) & & & & $0.072,0.995$ \\
\hline $40-49$ & 295 & $142(18.7)$ & $153(18.8)$ & \\
\hline$>49$ & 483 & $232(30.6)$ & $251(30.8)$ & \\
\hline Monthly income (RMB) & & & & $0.18,0.67$ \\
\hline$\leq 1500$ & 544 & $258(34.0)$ & $286(35.0)$ & \\
\hline $\begin{array}{l}\text { Opportunistic oral } \\
\text { infection }\end{array}$ & 66 & $35(4.6)$ & $31(3.8)$ & \\
\hline Education & & & & $0.036,0.982$ \\
\hline $\begin{array}{l}\text { Illiteracy and primary } \\
\text { school }\end{array}$ & 362 & $173(22.8)$ & 189 (23.2) & \\
\hline High school & 700 & $337(44.5)$ & $363(44.5)$ & \\
\hline College and above & 512 & $248(32.7)$ & $264(32.4)$ & \\
\hline
\end{tabular}




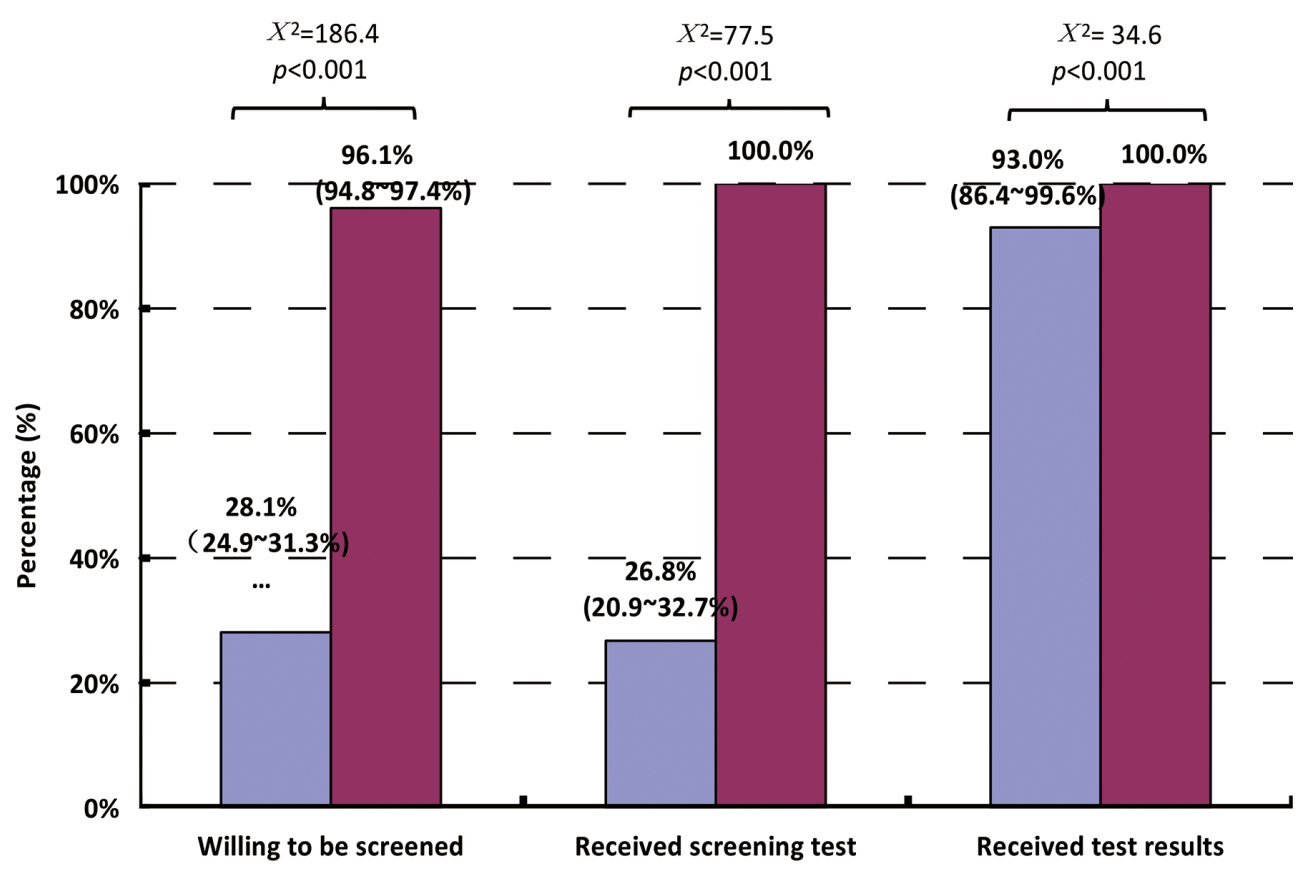

$\square$ Routine PITC

$\square$ Rapid PITC

Figure 2 Comparison of rates of 'willing to be screened', 'received screening test' and 'received test results' between routine and oral rapid testing groups. PITC, provider-initiated HIV testing and counselling.

the routine group patients completed the same process $(\chi 2=55.22, \mathrm{p}<0.01)$.

A total of 545 and 32 patients in routine and oral rapid testing groups provided reasons for not receiving HIV testing. The pattern varied substantially. Nearly half of the patients who did not wish to participate in routine PITC HIV testing cited 'HIV screening is too much hassle' $(254 / 545,46.6 \%$, figure 3$)$ as the main reason for refusal. This was followed by having had received screening previously $(124 / 545,22.8 \%)$ and self-perception of no risk of HIV infection $(103 / 545,18.9 \%)$. In contrast, among the 32 patients who refused oral rapid testing, $14(43.8 \%)$ indicated that they had been screened previously. This was followed by 'considering the test as offensive' $(13 / 32,40.6 \%)$ and self-perception of no risk of HIV infection $(3 / 32,9.4 \%)$. Only two regarded HIV screening as expensive (6.3\%). Notably, a small but substantial proportion of routine group participants

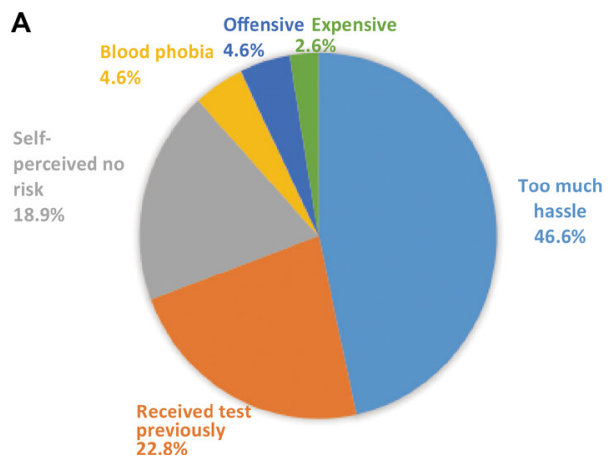

(25/545, 4.6\%) refused HIV testing owing to blood phobia. In contrast, none of the oral rapid testing group participants indicated this. However, when asked if they would be willing to pay $80 \mathrm{RMB}$ for an oral rapid HIV test in the rapid group, only 103/764 (13.5\%) patients answered "yes".

None of the 57 patients who completed blood testing in the routine group received a positive result. Seven of the 784 patients who completed oral rapid testing received positive results, but only three were found to be HIV positive in the following confirmation test. This corresponds to an HIV prevalence of $0.38 \%$ (3/784). This prevalence was not significantly different from that in the routine group $(\mathrm{p}=0.81)$. Among these infected patients, one had oral leukoplakia, and CD4+ T cell counts were 175 cells $/ \mu \mathrm{L}$, one had pulpitis with CD4+ $\mathrm{T}$ cell counts 453 cells $/ \mu \mathrm{L}$ and one had oral candidiasis with CD4+ T cell counts 36 cells $/ \mu \mathrm{L}$. Of the remaining four patients

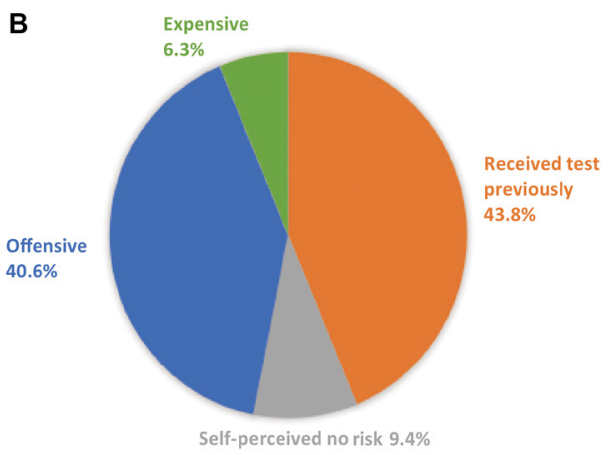

Figure 3 Reasons for refusing HIV testing among (A) 545 participants in routine provider-initiated HIV testing and counselling (PITC) and (B) 32 participants in oral rapid testing groups. 
with false-positive results, two had oral cancer, one had gingival bleeding, and one had an oral bacterial infection.

\section{DISCUSSION}

This study indicated that oral rapid HIV testing outperforms the routine PITC procedures and has a much better acceptance rate, HIV testing completion rate and results receiving rate. Most people who refused routine PITC testing did so because of blood phobia and the time needed. Oral rapid testing offers a non-invasive and time-effective approach to reach a much larger population.

\section{Strength of oral rapid HIV testing}

The superior result of oral rapid testing may be attributed to its prompt and simplified procedures. Unlike conventional routine HIV testing, oral rapid testing does not collect full blood samples, minimising safety concerns about the risk of needle exposure. Similarly, it also substantially reduces blood phobia in the participants. ${ }^{24}$ Further, it offers a much faster turnover for test results $(30 \mathrm{~min}$, compared with over a day for routine PITC testing) and flexibility for implementation during the waiting time before doctor consultation. In contrast, some patients in the routine group completed the test but did not return for the results. This might be owing to an inability to return on a separate day once they had returned to their busy life schedules. The quick availability of test results from an oral rapid test improved the willingness of the patients to wait.

Oral rapid HIV testing is easy to administer and implement. As the procedure does not rely on specialised equipment, such as microplate readers, centrifuges and refrigerated reagents, a private room and a health worker with appropriate training (eg, a nurse) are sufficient for implementing it $^{24}$; thereby it has significant advantages in outpatient clinic settings. Oral rapid HIV testing reduces the workload and demand of medical staff for blood collection and laboratory diagnosis, in turn giving them more opportunities to provide HIV counselling and establish a trust relationship with the patients. Past research has shown that nurse-initiated rapid testing is twice as acceptable as standard serum-based testing, leading to significantly higher awareness of test results. ${ }^{38} 39$ Consistently, our study showed a general acceptance of oral rapid HIV testing, which was conducted in a private setting and with test results notified individually. HIV counselling and status notification after testing reduces the risk of patient loss and facilitates their stay in care if they are diagnosed as HIV positive.

\section{Weaknesses of oral rapid HIV testing}

Oral rapid HIV testing may be less sensitive than the conventional serum-based approach $\left(2 \%\right.$ lower $\left.^{40}\right)$. In our study, we also reported four cases of false-positive results in oral rapid HIV testing. However, there have been conflicting reports about this. Some studies reported both sensitivity and specificity of oral rapid HIV testing to be $>99 \%$, whereas others suggested higher false-positive rates in oral rapid tests. ${ }^{4-44}$ Factors contributing to false positivity include errors in test performance and conduction, interpretation of results and suboptimum training of health workers. ${ }^{45}$ Among the four patients with a false-positive diagnosis, two had oral cancer one was pregnant and one had an oral bacterial infection. It is possible that the presence of oral cancer might bias the accuracy of oral rapid HIV testing, and further investigation is necessary to confirm this. Notably, two of the HIV-infected patients screened with oral rapid testing were found have CD4+ $\mathrm{T}$ cell counts that were much lower ( 36 and 175 cells $/ \mu \mathrm{L}$, respectively) than the current treatment criteria in China (500 cells $/ \mu \mathrm{L}$ ), highlighting the importance and urgency for scaling-up HIV testing in the general population.

HIV screening is provided free of charge only at voluntary counselling and testing sites, such as local Centers for Disease Control and HIV sentinel sites. At the study site, People's Hospital of Yuxi Prefecture, patients were required to pay 20 RMB for a conventional PITC test, and most patients can afford the fee if they decide to be tested. In contrast, only a few patients were willing to pay $80 \mathrm{RMB}$ for an oral rapid HIV test, as indicated in our study. The price of oral rapid HIV tests needs to be substantially reduced before it can be widely accepted and used in a clinical setting.

\section{Reasons for refusing HIV testing}

In the routine PITC group, nearly three-quarters of patients rejected HIV testing. The main barrier is the perception of tedious testing procedure and long waiting time, as nearly half of the people who refused testing regard it as 'too much hassle'. In contrast, the study of Jain et al, conducted in the emergency department in the USA, revealed only a $25.8 \%$ decline rate in HIV testing and the primary reason for declining was being recently tested with a negative result $(46 \%)$. Other studies in other settings also consistently showed similar decline rates $(2 \%-20 \%),{ }^{46-48}$ and in addition to citing having had a recent negative HIV test as the reason for the decline, self-perception of low risk for HIV infection, ${ }^{49}$ fear, self-denial and stigma also played a substantial role. ${ }^{17}{ }^{50}$ Our results appear to suggest that simplifying the HIV testing procedure alone would improve HIV testing acceptance significantly, as demonstrated in the oral rapid HIV testing group.

\section{Strengths and limitations of this study}

This is the first study in a less-developed Chinese setting to evaluate oral rapid HIV testing in clinical practices in comparison with routine oral HIV testing. The finding also illustrates the specific reasons for declining HIV testing.

Several limitations of the study should be noted. First, there may be a potential selection bias as participants were recruited primarily during weekday daytime hours and the research was conducted at two different periods of the same year. Patients who come at such a time might 
be more reluctant to participate than people who come at night or at weekends and patients in different months may vary. However, the two populations had no significant differences in demographic characteristics, and we selected the same doctors, nurses and venue for both processes to ensure the lowest bias effects. Second, participants might feel 'obligated' to participate in the study when they had been recommended to attend by the doctor, to avoid upsetting the clinicians. Third, participating nurses and doctors appeared to become more competent in the study during the oral rapid testing phase than in the earlier routine testing phase. But we did not assess how carefully nurses and dentists complied with the study protocol throughout the two phases. Fourth, although the study sample is representative of the local general population in Southwest China, the findings might not be generalisable to the whole country.

\section{CONCLUSIONS}

Oral rapid HIV testing demonstrated significantly higher screening acceptance, completion and test result return rate than routine serum-based HIV testing. Although low sensitivity remains a weakness, it overcomes the perceived complex procedure in routine testing and offers a feasible alternative for scaling-up HIV testing in the general Chinese population.

\section{Author affiliations}

'Division of HIV/AIDS and STI Control, Centers for Disease Control and Prevention, Yuxi Prefecture, Yunnan, China

${ }^{2}$ School of Public Health and Preventive Medicine, Faculty of Medicine, Nursing and Health Sciences, Monash University, Melbourne, VIC, Australia

${ }^{3}$ Research Center for Public Health, School of Medicine, Tsinghua University, Beijing, China

${ }^{4}$ Department of Stomatology, The First People's Hospital of Yuxi, Yuxi Prefecture, Yunnan, China

${ }^{5}$ Central Clinical School, Faculty of Medicine, Nursing and Health Sciences, Monash University, Melbourne, VIC, Australia

${ }^{6}$ Melbourne Sexual Health Centre, Alfred Health, Melbourne, Victoria, Australia

Acknowledgements We thank the patients who participated in this study, and all the doctors and nurses in the dental clinic outpatients in the Yuxi People's Hospital.

Contributors LZ, LC, JJ and FC conceived and designed the study; SL, SL, LG, YC, JF, CG and WL collected the data; SL cleaned the data; SL, SS and LZ analyzed the data; SL wrote the paper; LC, SS and LZ revised the manuscript. All authors approved the final manuscript.

Competing interests None declared.

Ethics approval Tsinghua University.

Provenance and peer review Not commissioned; externally peer reviewed.

Data sharing statement Due to privacy and ethical concerns, supporting data cannot be made openly available. Please contact the authors for the access of the original data.

Open Access This is an Open Access article distributed in accordance with the Creative Commons Attribution Non Commercial (CC BY-NC 4.0) license, which permits others to distribute, remix, adapt, build upon this work non-commercially, and license their derivative works on different terms, provided the original work is properly cited and the use is non-commercial. See: http://creativecommons.org/ licenses/by-nc/4.0/

(c) Article author(s) (or their employer(s) unless otherwise stated in the text of the article) 2017. All rights reserved. No commercial use is permitted unless otherwise expressly granted.

\section{REFERENCES}

1. Cui Y, Guo W, Li D, et al. Estimating HIV incidence among key affected populations in China from serial cross-sectional surveys in 2010-2014. J Int AIDS Soc 2016;19:206-9.

2. Lu L, Jia M, Ma Y, et al. The changing face of HIV in China. Nature 2008;455:609-11.

3. Centers for Disease Control and Prevention (CDC). Recommendations for HIV testing services for inpatients and outpatients in acute-care hospital settings. MMWR Morb Mortal Wkly Rep 1993;42:157-8.

4. Obermeyer CM, Osborn M. The utilization of testing and counseling for HIV: a review of the social and behavioral evidence. Am J Public Health 2007;97:1762-74.

5. Leon N, Naidoo P, Mathews C, et al. The impact of provider-initiated (opt-out) HIV testing and counseling of patients with sexually transmitted infection in Cape Town, South Africa: a controlled trial. Implement Sci 2010;5:8.

6. Sabin M, Lo YR. Progress in providing HIV testing and counseling in health facilities: WHO/UNAIDS guidance. JAMA 2010;304:342-3.

7. Chou R, Huffman LH, Fu R, et al; US Preventive Services Task Force. Screening for HIV: a review of the evidence for the U.S. preventive services task force. Ann Intern Med 2005;143:55-73.

8. Prevention CfDCa. HIV prevention through early detection and treatment of other sexually transmitted diseases--united states. recommendations of the advisory committee for HIV and STD prevention. MMWR Recomm Rep 1998;47:1-24.

9. Rotheram-Borus MJ, Newman PA, Etzel MA. Effective detection of HIV. J Acquir Immune Defic Syndr 2000;25:S105-S114.

10. Crepaz N, Lyles CM, Wolitski RJ, et al; HIV/AIDS Prevention Research Synthesis (PRS) Team. Do prevention interventions reduce HIV risk behaviours among people living with HIV? A meta-analytic review of controlled trials. AIDS 2006;20:143-57.

11. Cartoux M, Meda N, Van de Perre P, et al. Acceptability of voluntary HIV testing by pregnant women in developing countries: an international survey. Ghent International Working Group on Motherto-Child Transmission of HIV. AIDS 1998;12:2489-93.

12. Goodman E, Chesney MA, Tipton AC. Relationship of optimism, knowledge, attitudes, and beliefs to use of HIV antibody testing by at-risk female adolescents. Psychosom Med 1995;57:541-6.

13. Rou K, Guan J, Wu Z, et al. Demographic and behavioral factors associated with HIV testing in China. J Acquir Immune Defic Syndr 2009;50:432-4.

14. Stein JA, Nyamathi A. Gender differences in behavioural and psychosocial predictors of HIV testing and return for test results in a high-risk population. AIDS Care 2000;12:343-56.

15. Maman S, King E. Changes in HIV testing policies and the implications for women. J Midwifery Womens Health 2008;53:195-201.

16. Gruskin S, Ahmed S, Ferguson L. Provider-initiated HIV testing and counseling in health facilities--what does this mean for the health and human rights of pregnant women? Dev World Bioeth 2008;8:23-32.

17. Heijman RL, Stolte IG, Thiesbrummel HF, et al. Opting out increases HIV testing in a large sexually transmitted infections outpatient clinic. Sex Transm Infect 2009;85:249-55.

18. Brown J, Kuo I, Bellows J, et al. Patient perceptions and acceptance of routine emergency department HIV testing. Public Health Rep 2008;123:21-6.

19. Shagufta Z, Mahto M. Uptake of HIV testing in a genitourinary medicine clinic following opt-out screening method and uptake rate by individual clinicians. Int J STD AIDS 2007;18:650-1.

20. Stanley B, Fraser J, Cox NH. Uptake of HIV screening in genitourinary medicine after change to "opt-out" consent. BMJ 2003;326:1174

21. Branson BM, Handsfield HH, Lampe MA, et al; Centers for Disease Control and Prevention (CDC). Revised recommendations for HIV testing of adults, adolescents, and pregnant women in health-care settings. MMWR Recomm Rep 2006;55:1-17. quiz CE1-4.

22. Njeru MK, Blystad A, Shayo EH, et al. Practicing provider-initiated HIV testing in high prevalence settings: consent concerns and missed preventive opportunities. BMC Health Serv Res 2011;11:87.

23. Siegel K, Abel SN, Pereyra M, et al. Rapid HIV testing in dental practices. Am J Public Health 2012;102:625-32.

24. Pant Pai N, Joshi R, Dogra S, et al. Evaluation of diagnostic accuracy, feasibility and client preference for rapid oral fluid-based diagnosis of HIV infection in rural India. PLoS One 2007;2:e367.

25. Dietz CA, Ablah E, Reznik D, et al. Patients' attitudes about rapid oral HIV screening in an urban, free dental clinic. AIDS Patient Care STDS 2008;22:205-12.

26. Kang CR, Bang JH, Cho SI, et al. Implementing the use of rapid HIV tests in public health centers in Seoul: results of a pilot project, 2014. J Korean Med Sci 2016;31:467-9. 
27. Flowers P, Riddell J, Park C, et al. Preparedness for use of the rapid result HIV self-test by gay men and other men who have sex with men (MSM): a mixed methods exploratory study among MSM and those involved in HIV prevention and care. HIV Med 2017;18:245-55.

28. Chan D, Stewart M, Smith M, et al. The rise of targeted HIV oral rapid testing in Australia. Med J Aust 2015;202:251-4.

29. Prost A, Griffiths CJ, Anderson J, et al. Feasibility and acceptability of offering rapid HIV tests to patients registering with primary care in London (UK): a pilot study. Sex Transm Infect 2009;85:326-9.

30. Ngaihte PC, Santellab AJ, Wattc RG, et al. Envisioning the role of indian dentists in conducting rapid HIV testing for early detection of HIV. International Journal of Development Research 2015;5:5975-7.

31. Marley G, Kang D, Wilson EC, et al. Introducing rapid oral-fluid HIV testing among high risk populations in Shandong, China: feasibility and challenges. BMC Public Health 2014;14:422.

32. Wang L, Santella AJ, Huang R, et al. Knowledge of HIV and willingness to conduct oral rapid HIV testing among dentists in Xi'an China. PLoS One 2015;10:e0119274.

33. Chow EP, Gao L, Chen L, et al. Shifting patterns of the HIV epidemic in southwest China: a case study based on sentinel surveillance, 1995-2012. AIDS Patient Care STDS 2015;29:314-20.

34. Wang B, Liang $Y$, Wang $Y$, et al. HIV prevalence and phylogenetic characteristics among entry travelers in Xishuangbanna Prefecture, Yunnan Province, between 2003 and 2012. J Med Virol 2016;1.

35. Huang M-B, Ye L, Liang B-Y, et al. Characterizing the HIV/AIDS epidemic in the United States and China. Int J Environ Res Public Health 2016;13:30

36. Xun $\mathrm{H}$, Kang D, Huang $\mathrm{T}$, et al. Factors associated with willingness to accept oral fluid HIV rapid testing among most-at-risk populations in China. PLoS One 2013;8:e80594.

37. Brondani M, Chang S, Donnelly L. Assessing patients' attitudes to opt-out HIV rapid screening in community dental clinics: a crosssectional canadian experience. BMC Res Notes 2016;9:264.

38. Anaya HD, Hoang T, Golden JF, et al. Improving HIV screening and receipt of results by nurse-initiated streamlined counseling and rapid testing. J Gen Intern Med 2008;23:800-7.

39. Jackson-Malik P, McLaughlin MJ, O'Hara KT, et al. Rapid oral fluid testing for HIV in veterans with mental health diagnoses and residing in community-assisted living facilities. J Assoc Nurses AIDS Care 2011;22:81-9.

40. Pant Pai N, Balram B, Shivkumar S, et al. Head-to-head comparison of accuracy of a rapid point-of-care HIV test with oral versus wholeblood specimens: a systematic review and meta-analysis. Lancet Infect Dis 2012;12:373-80.

41. Piwowar-Manning EM, Tustin NB, Sikateyo $P$, et al. Validation of rapid HIV antibody tests in 5 African countries. J Int Assoc Physicians AIDS Care 2010;9:170-2.

42. Pascoe SJ, Langhaug LF, Mudzori J, et al. Field evaluation of diagnostic accuracy of an oral fluid rapid test for HIV, tested at pointof-service sites in rural Zimbabwe. AIDS Patient Care STDS 2009;23:571-6.

43. Walensky RP, Arbelaez C, Reichmann WM, et al. Revising expectations from rapid HIV tests in the emergency department. Ann Intern Med 2008;149:153-60.

44. Stekler JD, Swenson PD, Coombs RW, et al. HIV testing in a highincidence population: is antibody testing alone good enough? Clin Infect Dis 2009;49:444-53.

45. Centers for Disease Control and Prevention (CDC). False-positive oral fluid rapid HIV tests--New York City, 2005-2008. MMWR Morb Mortal Wkly Rep 2008;57:660-5.

46. Waxman MJ, Kimaiyo S, Ongaro N, et al. Initial outcomes of an emergency department rapid HIV testing program in western Kenya. AIDS Patient Care STDS 2007;21:981-6.

47. Kassler WJ, Dillon BA, Haley C, et al. On-site, rapid HIV testing with same-day results and counseling. AIDS 1997;11:1045-51.

48. Donnell-Fink L, Reichmann WM, Arbelaez C, et al. Patient satisfaction with rapid HIV testing in the emergency department. Ann Emerg Med 2011;58:S49-S52.

49. Jain S, Lowman ES, Kessler A, et al. Seroprevalence study using ora rapid HIV testing in a large urban emergency department. J Emerg Med 2012;43:e269-e275.

50. Batey DS, Hogan VL, Cantor R, et al. Short communication routine HIV testing in the emergency department: assessment of patient perceptions. AIDS Res Hum Retroviruses 2012;28:352-6. 